\title{
RECEPTOR ANTAGONISTS
}

\section{The search for calcium receptor antagonists (calcilytics)}

\author{
E F Nemeth \\ NPS Pharmaceuticals Inc., 30 College Street/Suite 301, Toronto, Ontario, Canada M5G 1K2 \\ (Requests for offprints should be addressed to E F Nemeth; Email: enemeth@npsp.com)
}

\begin{abstract}
The $\mathrm{Ca}^{2+}$ receptor on the surface of parathyroid cells is the primary molecular entity regulating secretion of parathyroid hormone (PTH). Because of this, it is a particularly appealing target for new drugs intended to increase or decrease circulating levels of PTH. Calcilytic compounds are $\mathrm{Ca}^{2+}$ receptor antagonists which increase the secretion of PTH. The first reported calcilytic compound was NPS 2143, an orally active molecule which elicits rapid, 3 - to 4 -fold increases in circulating levels of PTH. These rapid changes in plasma PTH levels are sufficient to increase bone turnover in ovariectomized, osteopenic rats. When administered together with an antiresorptive agent (estradiol), NPS 2143 causes an increase in trabecular bone volume and bone mineral density in osteopenic rats. The magnitude of these changes are far in excess of those caused by estradiol alone and are comparable with those achieved by daily administration of PTH or a peptide analog. These anabolic effects of NPS 2143 on bone are not associated with hyperplasia of the parathyroid glands. Calcilytic compounds can increase endogenous levels of circulating PTH to an extent that stimulates new bone formation. Such compounds could replace the use of exogenous PTH or its peptide fragments in treating osteoporosis.
\end{abstract}

Journal of Molecular Endocrinology (2002) 29, 15-21

\section{Introduction}

Parathyroid hormone (PTH) is arguably the most important endocrine factor regulating systemic $\mathrm{Ca}^{2+}$ homeostasis. PTH acts on target cells in both the kidney and the skeleton to increase plasma levels of $\mathrm{Ca}^{2+}$. Within the skeleton, PTH increases bone turnover but the resulting overall effect on bone is highly dependent on temporal changes in the circulating levels of PTH. Thus, sustained elevations in plasma PTH levels, as occur in primary or secondary hyperparathyroidism, have a net catabolic effect on the skeleton (Antonsen \& Sherrard 1995). In contrast, temporary increases in plasma levels of PTH achieved by the daily (or near daily) injection of exogenous hormone, have a net anabolic effect on the skeleton (Dempster et al. 1993, Seeman \& Delmas 2001). The profound stimulatory effect on bone formation has generated interest in the use of PTH or its biologically active peptide analogs as first generation anabolic therapies for osteoporosis.

The need for such an anabolic therapy is underscored by the serious health problem posed by osteoporosis, the incidence of which is increasing as the general population ages. While currently available antiresorptive therapies, such as estrogens or bisphosphonates, prevent further bone loss, they cause relatively small increases in new bone formation. The ability to stimulate new bone formation and thereby replace bone already lost to disease would constitute a significant advance in the treatment of osteoporosis. A first step in this direction is teraparatide, a peptide fragment of PTH which has recently been recommended for approval in the US for treating severe osteoporosis (Fox 2002). Human PTH is in phase III clinical trials and other peptide fragments of PTH are in earlier stages of development. However, the therapeutic usefulness of all these 
peptides is compromised by the need for systemic administration of a costly biological agent.

An alternative approach which might overcome these drawbacks, and yet achieve similar anabolic effects on bone, is based on the use of orally active compounds which block the activity of the parathyroid $\mathrm{Ca}^{2+}$ receptor and stimulate secretion of endogenous PTH (Nemeth 2002). Compounds with the appropriate pharmacokinetic profile would be expected to cause a marked but transient increase in circulating levels of PTH, sufficient to stimulate new bone formation. With this goal in mind, we initiated a drug discovery program in collaboration with SmithKline Beecham (now GlaxoSmithKline) in 1993. This Commentary is a snapshot of a work in progress which captures some of the challenges encountered in this novel approach to treating osteoporosis.

\section{Trying to inhibit $\mathrm{Ca}^{2+}$ receptor activity}

The $\mathrm{Ca}^{2+}$ receptor is a $\mathrm{G}$ protein-coupled receptor that shares structural features with the metabotropic glutamate receptors (mGluRs) and the $\gamma$-aminobutyric acid type $B$ receptors $\left(\mathrm{GABA}_{\mathrm{B}} \mathrm{Rs}\right)$ (see Brown \& MacLeod 2001). All these receptors possess a large extracellular domain which is believed to bind the cognate physiological ligand glutamate, $\gamma$-aminobutyric acid or extracellular $\mathrm{Ca}^{2+}$ (Hammerland et al. 1999). At present, only one gene coding for the $\mathrm{Ca}^{2+}$ receptor has been identified; no receptor subtypes have been found and splice variants of the $\mathrm{Ca}^{2+}$ receptor are few and of low abundance (Garrett et al. 1995). The parathyroid $\mathrm{Ca}^{2+}$ receptor couples through a $\mathrm{G}_{\mathrm{i}}$ protein to adenylate cyclase and through a $\mathrm{G}_{\mathrm{q}} / \mathrm{G}_{11}$ protein to phospholipase $\mathrm{C}$. Activation of the parathyroid cell $\mathrm{Ca}^{2+}$ receptor by increased levels of extracellular $\mathrm{Ca}^{2+}$ causes a slight decrease in cAMP levels, a profound increase in the concentration of cytoplasmic $\mathrm{Ca}^{2+}\left(\left[\mathrm{Ca}^{2+}\right]_{\mathrm{i}}\right)$ and a decrease in the secretion of PTH. In its structural and functional properties, the $\mathrm{Ca}^{2+}$ receptor is akin to other $\mathrm{G}$ protein-coupled receptors which transduce an extracellular signal into a functional cellular response. The unique difference is that the primary physiological ligand for the $\mathrm{Ca}^{2+}$ receptor is an inorganic ion rather than an organic molecule.

The search for $\mathrm{Ca}^{2+}$ receptor ligands began shortly after I convinced myself (and few others at the time!) that such a receptor existed (Nemeth \& Scarpa 1986). It soon became clear that this putative $\mathrm{Ca}^{2+}$ receptor was rather promiscuous and could be activated by a variety of di- and trivalent cations and many structurally diverse organic compounds which share the common property of possessing a net positive charge at physiological $\mathrm{pH}$. Included in the category of molecular ligands are polyamines from mammals and the venoms of spiders, aminoglycoside antibiotics, and poly-basic amino acids. The response to extracellular $\mathrm{Ca}^{2+}$ and all the organic and inorganic polycations was potentiated by certain phenylalkylamines like verapamil or the trimethoxybenzoate derivative TMB-8. All these substances have now been shown to act directly on the $\mathrm{Ca}^{2+}$ receptor. Ligands which mimic or potentiate the actions of extracellular $\mathrm{Ca}^{2+}$ at the $\mathrm{Ca}^{2+}$ receptor have been termed calcimimetics (Nemeth et al. 1998). Calcimimetics can be either inorganic ions or organic molecules and they can act as agonists (type I) or allosteric activators (type II) of the receptor. The phenylalkylamines are examples of type II calcimimetics whereas all the inorganic and organic polycations are type I calcimimetic ligands. Calcimimetic compounds inhibit the secretion of PTH and lower circulating levels of PTH. A type II calcimimetic compound is now in phase III clinical trials for the treatment of hyperparathyroidism (Nemeth 2002).

All this pharmacology regarding agonists and allosteric activators was established before the $\mathrm{Ca}^{2+}$ receptor was cloned and structurally characterized (in February 1993, Brown et al. (1993)). Yet it is curious that during all this testing we never discovered any ligand capable of blocking $\mathrm{Ca}^{2+}$ receptor activity. This is unusual for $\mathrm{G}$ proteincoupled receptors because it has traditionally been much easier to find antagonists of these receptors rather than agonists or allosteric activators. The $\mathrm{Ca}^{2+}$ receptor is somehow different, and calcimimetic ligands appear to be rather plentiful. There is seemingly some intrinsic feature of the $\mathrm{Ca}^{2+}$ receptor, or of the manner in which it couples to effector systems, which makes its activity difficult to suppress. In any event, this feature of $\mathrm{Ca}^{2+}$ receptor biology has made the quest for antagonists considerably more laborious than the corresponding search for agonists.

In searching for antagonists of $\mathrm{G}$ protein-coupled receptors, one typically starts with structural modifications to the cognate physiological agonist 
in the hopes that a high affinity ligand lacking intrinsic efficacy will be created. The physiological agonist of the $\mathrm{Ca}^{2+}$ receptor, however, does not lend itself to the usual tricks of the medicinal chemist. And all the structural modifications made to molecular calcimimetics like NPS R-568 simply yielded more calcimimetics. Molecular modeling of the receptor and its presumed binding pocket for molecular calcimimetics was likewise unproductive. Given this vacuum of structural information, we had to rely on random screening of compound libraries. Initially, bovine parathyroid cells were used since there are no cell lines which retain the appropriate parathyroid phenotype. Once the $\mathrm{Ca}^{2+}$ receptor was cloned, however, we were able to construct a stably transfected cell line and initiate high-throughput screening. Doing this for a number of years reinforced the original suspicion that it is difficult to block $\mathrm{Ca}^{2+}$ receptor activity. But it is not impossible.

Eventually, a compound that did block $\mathrm{Ca}^{2+}$ receptor activity was discovered. The initial compound detected by high-throughput screening was far from potent and lacked specificity. In fact, it was four orders of magnitude more potent on adrenergic receptors than on the $\mathrm{Ca}^{2+}$ receptor. Yet this initial compound was not pan active. It did not, for example, inhibit the activity of many other $\mathrm{G}$ protein-coupled receptors upon which it was tested. And it did have drug-like characteristics which attracted the attention of the medicinal chemists. Their persistence and expertise was rewarded with a series of compounds with greatly improved potency and selectivity at the $\mathrm{Ca}^{2+}$ receptor. The compounds emerging from this effort are the first substances, either atomic or molecular, shown to block $\mathrm{Ca}^{2+}$ receptor activity. Ligands which block the activity of the $\mathrm{Ca}^{2+}$ receptor have been termed calcilytics (Nemeth et al. 2001). Together with certain calcimimetic molecules, these compounds are valuable tools to determine the feasibility of developing drugs which target the $\mathrm{Ca}^{2+}$ receptor for various bone and mineral related disorders.

One of the early calcilytic compounds studied in some detail is NPS 2143 (Fig. 1). The structure of this compound illustrates some basic features of the first generation calcilytic pharmacophore. The stereochemistry of the hydroxyl group is critical and $R$-enantiomers are more potent - a fortunate outcome since potency on adrenergic receptors tracks $S$-enantiomers. Replacement of the gemdimethyl with hydrogen or methyl results in a lose of potency. The alkyl spacing between these moieties and the right and left hand aromatic portions of the molecule are also important. The left and right hand aromatic regions of the molecule are somewhat more tolerant of structural diversity but loss of the cyano group greatly diminishes potency.

As detailed below, NPS 2143 possesses sufficient potency and selectivity to be used as a tool compound. While not perfect, it served the purpose of testing some of our early concerns regarding the technical feasibility of blocking the $\mathrm{Ca}^{2+}$ receptor to stimulate PTH secretion and new bone formation.

\section{Proof of concept with a prototype calcilytic compound}

From the outset, there were a number of uncertainties which challenged the calcilytic approach to treating osteoporosis. Indeed, the fundamental hypothesis supporting this approach to building bone was suspect; because there was no ligand which inhibited the $\mathrm{Ca}^{2+}$ receptor, there was no reason to suppose that blocking the activity of the $\mathrm{Ca}^{2+}$ receptor would, in fact, stimulate PTH secretion. Although hypocalcemia stimulates PTH secretion, this is not necessarily equivalent to blocking $\mathrm{Ca}^{2+}$ receptor activity in the setting of normocalcemia. More worrisome was the possibility that PTH could not be secreted from the parathyroid glands in sufficient quantities to stimulate new bone formation. And finally, would blocking the $\mathrm{Ca}^{2+}$ receptor on a daily basis be perceived by the parathyroid glands as hypocalcemia, and possibly trigger cellular hyperplasia leading to chronically elevated levels of PTH (and net bone loss!)?

NPS 2143 was selected as a tool compound to address these issues. This compound inhibits extracellular $\mathrm{Ca}^{2+}$-evoked increases in $\left[\mathrm{Ca}^{2+}\right]_{\mathrm{i}}$ in HEK 293 cells expressing the human $\mathrm{Ca}^{2+}$ receptor $\left(\mathrm{IC}_{50}=43 \mathrm{nM}\right)$ and stimulates PTH secretion from bovine parathyroid cells in vitro $\left(\mathrm{EC}_{50}=41 \mathrm{nM}\right.$; Fig. 1). The inhibitory effects of NPS 2143 are not dependent on the nature of the ligand used to activate the $\mathrm{Ca}^{2+}$ receptor and this compound has similar potencies on responses 
<smiles>CC(C)(Cc1ccc2ccccc2c1)NC[C@@H](O)COc1cccc(Cl)c1C#N</smiles>

NPS 2143

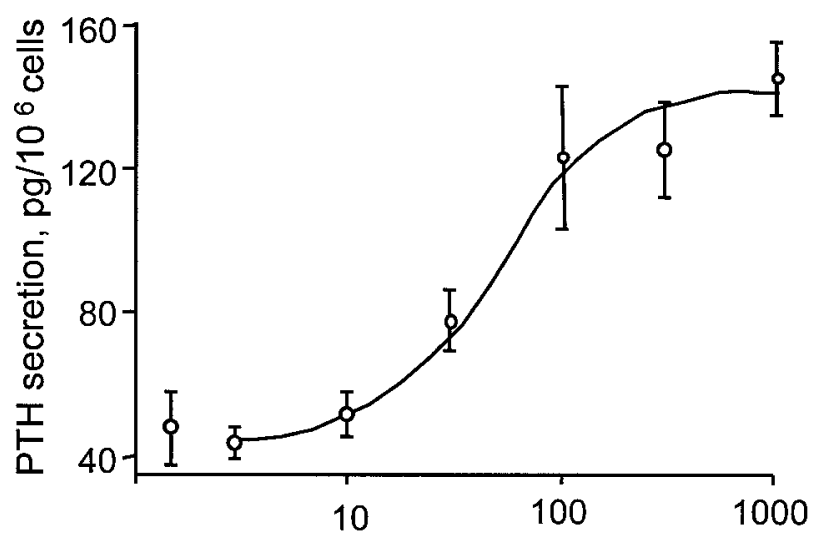

NPS 2143, nmol/L

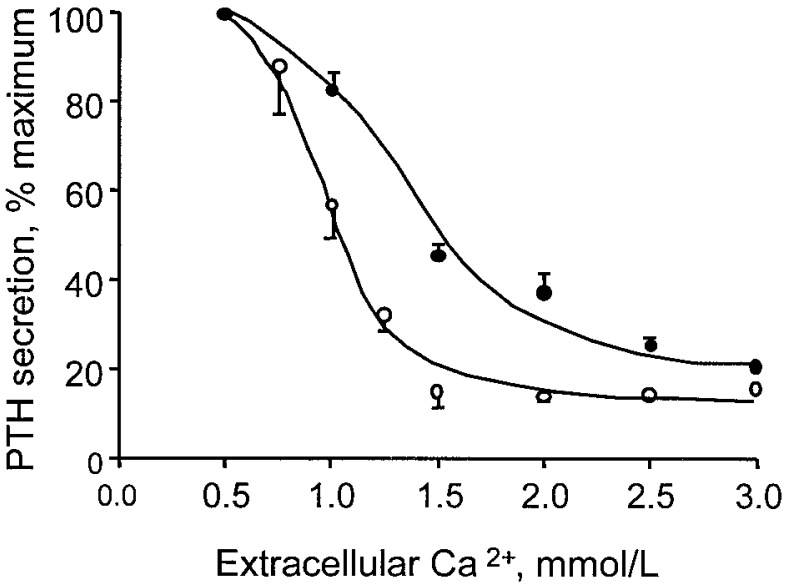

Figure 1. Structure of the calcilytic compound NPS 2143. Left panel: NPS 2143 stimulates PTH secretion from bovine parathyroid cells in vitro. Right panel: parathyroid cells were incubated in the presence $(\bullet)$ or absence $(0)$ of 300 nM NPS 2143 and the indicated concentration of extracellular $\mathrm{Ca}^{2+}$. (Fig. 7 in Nemeth et al. 2001.)

elicited by type I or type II calcimimetics. Calcilytic compounds with this pharmacophore appear to act by an allosteric mechanism which shifts the concentration-response curve for extracellular $\mathrm{Ca}^{2+}$ to the right without affecting maximal or minimal responses (Fig. 1). In this respect, these compounds act like type II calcimimetics, only they decrease, rather increase, the sensitivity of the $\mathrm{Ca}^{2+}$ receptor to activation by extracellular $\mathrm{Ca}^{2+}$. This mechanism of action suggests that the maximal effect of this compound in vivo will occur under normocalcemic conditions.

When administered to rats either orally or by i.v. infusion, NPS 2143 causes a prompt increase in circulating levels of $\mathrm{PTH}$, the magnitude of which is dose-dependent (Fig. 2). These findings were comforting because, as noted above, it was never a certainty that a calcilytic compounds would in fact stimulate PTH secretion. Moreover, the magnitude of the increase in circulating levels of endogenous
PTH is similar to that achieved by exogenous peptide when administered at doses which stimulate new bone formation (Fox et al. 1997).

NPS 2143 possesses reasonable selectivity at concentrations below $1 \mu \mathrm{M}$. It does not, for example, affect the activity of receptors which are most homologous to the $\mathrm{Ca}^{2+}$ receptor such as various mGluRs or the two $\mathrm{GABA}_{\mathrm{B}} \mathrm{Rs}_{\mathrm{s}}$ (Nemeth et al. 2001). When studied in rats, the compound stimulated tubular reabsorption of $\mathrm{Ca}^{2+}$, an effect that might result from a direct action on renal $\mathrm{Ca}^{2+}$ receptors or be indirectly mediated by an increase in plasma PTH levels. This compound is not, however, without its blemishes, and its pharmacokinetic profile is less than ideal. To achieve an anabolic effect on bone, the increase in circulating levels of PTH must be transient. Although the boundaries of 'transient' have not been rigorously explored, it is generally accepted that a 3- to 4-fold increase in plasma PTH levels lasting 1-2 h would 

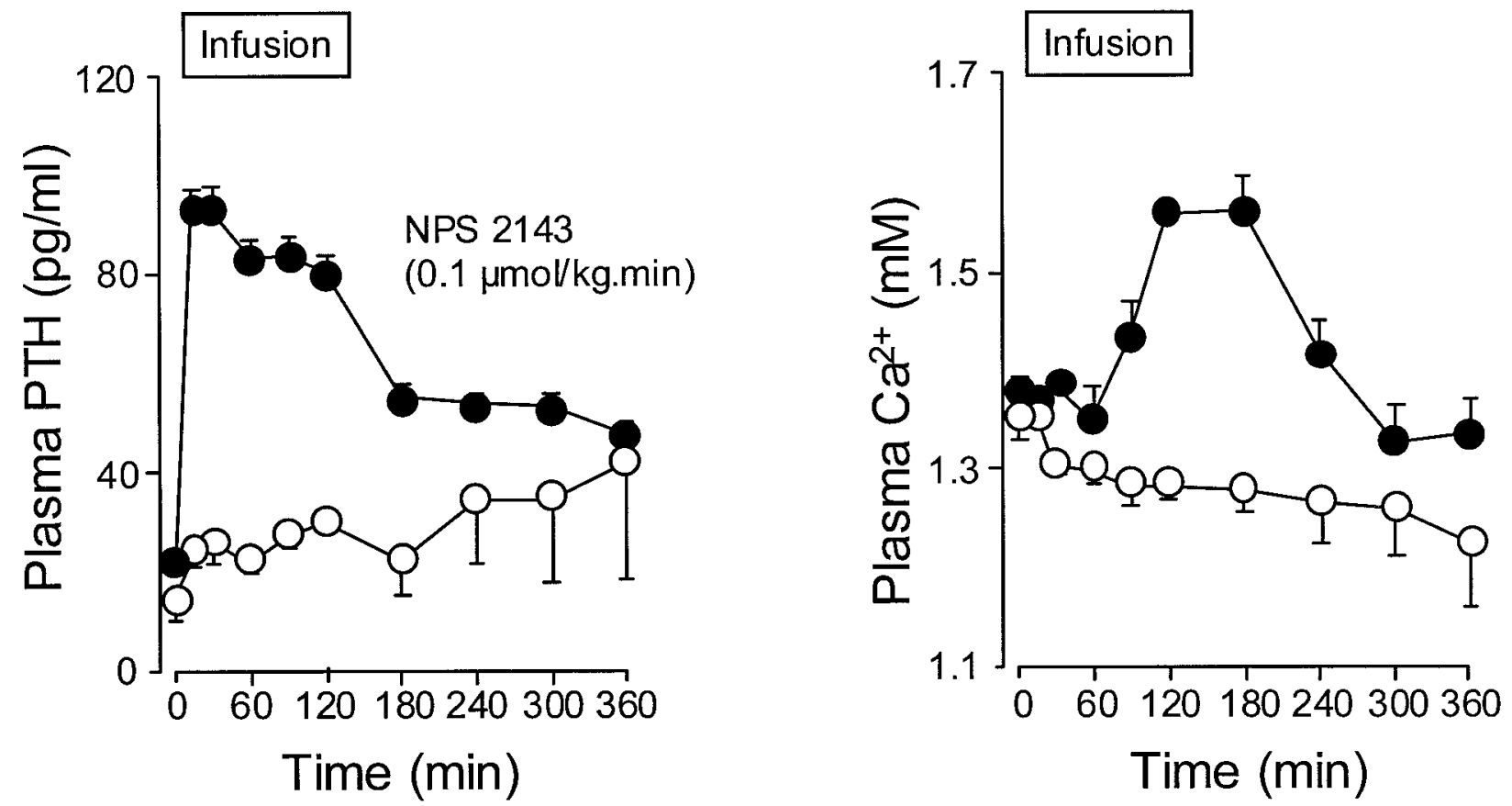

Figure 2 NPS 2143 rapidly increases circulating levels of PTH in normal rats. NPS 2143 was infused i.v. $(0.1 \mu \mathrm{mol} / \mathrm{kg}$ per $\mathrm{min})(\bullet)$ for $2 \mathrm{~h}$. (Fig. 9 in Nemeth et al. 2001.)

be required to stimulate new bone formation without activating resorption resulting in a net anabolic effect on the skeleton (Hodsman et al. 2002). Thus, a short acting calcilytic is desired. The compound must be orally active and rapidly absorbed and the compound (and any active metabolite) must be rapidly cleared from the body once it is in the systemic circulation. NPS 2143 is not rapidly eliminated from the body following oral administration and it causes a sustained, rather than transient increase in circulating levels of PTH (Gowen et al. 2000). Nonetheless, this compound can still be used to determine if the magnitude of the increase in plasma levels of PTH is sufficient to stimulate new bone formation.

The effects of NPS 2143 on circulating levels of PTH and bone turnover were assessed in ovariectomized rats. Daily oral administration of NPS 2143 began 3 months after ovariectomy and continued for 5 weeks at which time static and dynamic parameters of bone histomorphometry were estimated. Plasma levels of PTH were elevated for at least $4 \mathrm{~h}$ following oral dosing with NPS 2143 but returned to baseline levels within $24 \mathrm{~h}$. Morphometric parameters indicative of bone resorption or bone formation were increased by
NPS 2143 and their magnitudes were comparable so there was no net increase in bone mineral density (BMD) in the distal femur or the proximal tibia (Gowen et al. 2000). This is exactly what would be expected from the chronically elevated levels of PTH and it demonstrates that blocking parathyroid $\mathrm{Ca}^{2+}$ receptor activity with a small molecule will release PTH in amounts which are sufficient to increase bone turnover. It seemed reasonable to suppose that blocking the increased bone resorption induced by NPS 2143 might isolate the mechanisms underlying bone formation and result in a net anabolic effect. Indeed, when the animals received the combination of NPS 2143 and the antiresorptive agent $17 \beta$-estradiol, there were increases in trabecular area and bone formation rate accompanied by net increases in BMD (Fig. 3). Thus, a calcilytic compound can result in a net anabolic effect on bone when administered together with a conventional antiresorptive.

The long half-life of NPS 2143 in the body also affords a stringent test of whether the parathyroid cell will perceive a calcilytic compound as a hypocalcemic stimulus and thereby undergo glandular hyperplasia and/or hypertrophy (Wada 


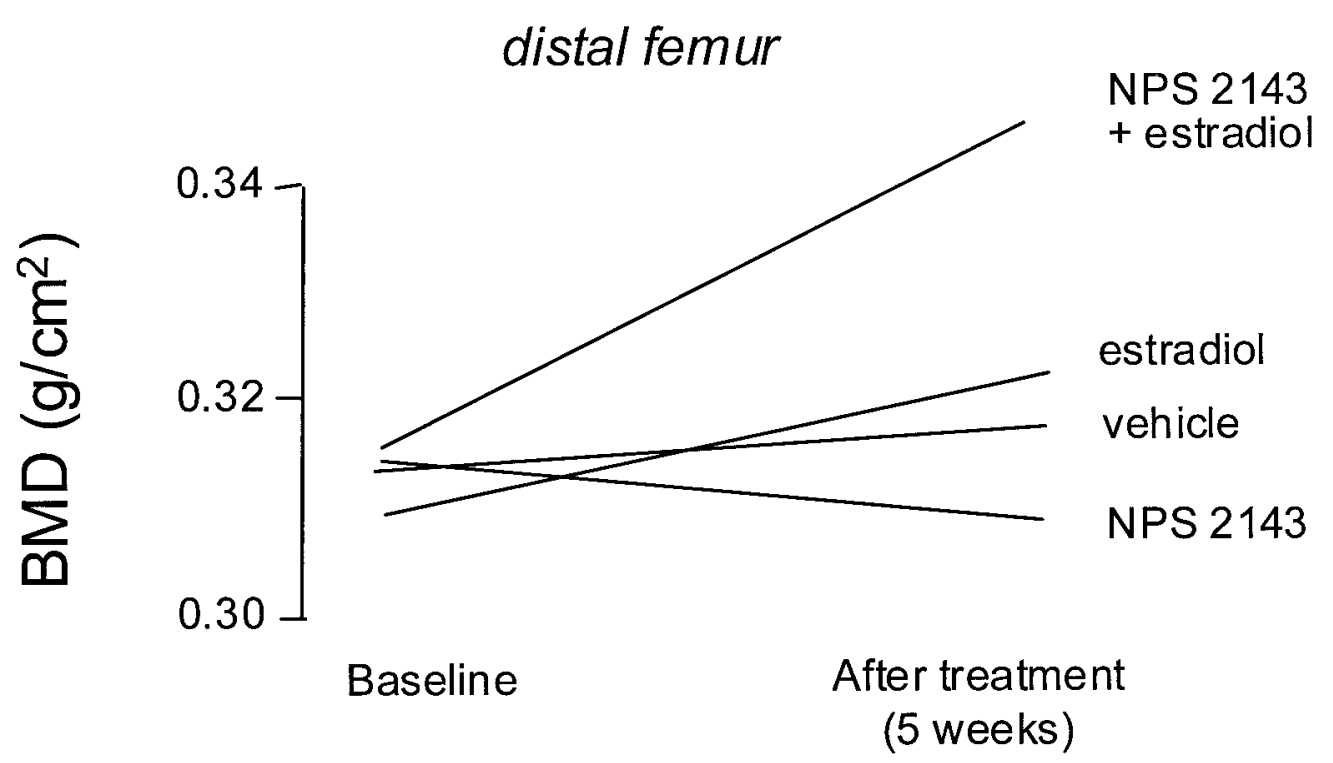

Figure 3. Treatment with NPS 2143 and estrogen increases BMD in the distal femur of ovariectomized rats. (Fig. 8 in Gowen et al. 2000.)

et al. 1997, Parfitt 2001). Cellular proliferation was assessed during the last week of dosing in the studies described above. When compared with sham or ovariectomized animals, NPS 2143 did not cause hyperplasia (as assessed by bromodeoxyuridine staining), whether given alone or in combination with $17 \beta$-estradiol (Gowen et al. 2000). Measurements of NPS 2143 in the plasma revealed that levels were still elevated $8 \mathrm{~h}$ after a single oral dose and this might be expected to provide a chronic hypocalcemic stimulus to the parathyroid glands, yet this did not result in cellular hyperplasia or hypotrophy. It is always possible that more prolonged treatment with a long-acting calcilytic compound might have triggered cellular proliferation in the parathyroid glands but the proliferative response to hypocalcemia occurs rapidly in the parathyroids (within a week). It seems that a short-acting calcilytic compound would be unlikely to stimulate parathyroid gland hyperplasia.

\section{Conclusion}

All the evidence obtained to date supports the view that the parathyroid $\mathrm{Ca}^{2+}$ receptor is a viable target for drugs which could have anabolic effects on bone. Although the prototype calcilytic compound NPS 2143 is not a clinical candidate, it has proven to be a valuable tool to explore the feasibility of targeting the $\mathrm{Ca}^{2+}$ receptor with antagonist compounds to increase circulating levels of PTH and thereby stimulate new bone formation. There are still some basic issues to resolve, however, and it cannot be stated with certainty that all the effects of calcilytic compounds on bone result from an action solely at the parathyroid $\mathrm{Ca}^{2+}$ receptor. $\mathrm{Ca}^{2+}$ receptors might be expressed on certain cells of bone and these would be expected to be targets for calcilytic compounds. So far, however, we have not noted any effects of calcilytic compounds on osteoblastic or osteoclastic activity in vitro. Perhaps the most challenging aspect of this new approach to treating osteoporosis will be achieving the necessary pharmacokinetic features of rapid absorption and elimination to cause a transient increase in circulating levels of PTH. A calcilytic compound with an improved phamacokinetic profile has recently entered phase I clinical trials so we will soon know if we can achieve this therapeutic goal, which we irreverently refer to as 'get in...get out...nobody gets hurt'.

\section{Acknowledgements}

This research has been a collaborative effort between scientists at GlaxoSmithKline and NPS Pharmaceuticals and many individuals have made (and continue to make) significant contributions to 
this program. Some of the key players who participated in the early work described here are, from GlaxoSmithKline: Maxine Gowen, George Stroup, Pradip Bhatnagar and Brian Smith; and from NPS: John Fox, Ric DelMar, Mike Miller, Rebecca Conklin, Alec Harootunian and Will Heaton.

\section{References}

Antonsen JE \& Sherrard DJ 1995 Renal osteodystrophy: past and present. Seminars in Dialysis 9 296-302.

Brown EM \& MacLeod RJ 2001 Extracellular calcium sensing and extracellular calcium signaling. Physiological Reviews 81 239-297.

Brown EM, Gamba G, Riccardi D, Lombardi M, Butters R, Kifor O, Sun A, Hediger MA, Lytton J \& Hebert SC 1993 Cloning and characterization of an extracellular $\mathrm{Ca}^{2+}$-sensing receptor from bovine parathyroid. Nature 366 575-580.

Dempster DW, Cosman F, Parisien M, Shen V \& Lindsay R 1993 Anabolic actions of parathyroid hormone on bone. Endocrine Reviewes 14 690-709.

Fox J 2002 Developments in parathyroid hormone and related peptides as bone-formation agents. Current Opinion in Pharmacology 2 338-344.

Fox J, Miller MA, Stroup GB, Nemeth EF \& Miller SC 1997 Plasma levels of parathyroid hormone that induce anabolic effects in bone ovariectomized rats can be achieved by stimulation of endogenous hormone secretion. Bone 21 163-169.

Garrett JE, Capuano IV, Hammerland LG, Hung BCP, Brown EM, Hebert SC, Nemeth EF \& Fuller F 1995 Molecular cloning and functional expression of human parathyroid calcium receptor cDNAs. Fournal of Biological Chemistry 270 12919-12925.

Gowen M, Stroup GB, Dodds RA, James IE, Votta BJ, Smith BR, Bhatnagar PK, Lago AM, Callahan JF, DelMar EG, Miller MA, Nemeth EF \& Fox J 2000 Antagonizing the parathyroid calcium receptor stimulates parathyroid hormone secretion and bone formation in osteopenic rats. Fournal of Clinical Investigation 105 1595-1604.

Hammerland LG, Krapcho KJ, Garrett JE, Alasti N, Hung BCP, Simin RT, Levinthal C, Nemeth EF \& Fuller FH 1999 Domains determining ligand specificity for $\mathrm{Ca}^{2+}$ receptors. Molecular Pharmacology 55 642-648.

Hodsman AB, Hanley DA, Watson PH \& Fraher LJ 2002 Parathyroid hormone. In Principles of Bone Biology, edn 2, pp 1305-1324. Eds JP Bilezikian, LG Raisz \& GA Rodan. San Diego, CA: Academic Press.

Nemeth EF 2002 Calcium receptors as novel drug targets. In Principles of Bone Biology, edn 2, pp 1339-1359. Eds JP Bilezikian, LG Raisz \& GA Rodan. San Diego, CA: Academic Press.

Nemeth EF \& Scarpa A 1986 Cytosolic $\mathrm{Ca}^{2+}$ and the regulation of secretion in parathyroid cells. FEBS Letters 203 15-19.

Nemeth EF, Steffey ME, Hammerland L, Hung BCP, Van Wagenen BC, DelMar EG \& Balandrin MF 1998 Calcimimetics with potent and selective activity on the parathyroid calcium receptor. PNAS 95 4040-4045.

Nemeth EF, DelMar EG, Heaton WL, Miller MA, Lambert LD, Conklin RL, Gowen M, Gleason JG, Bhatnagar PK \& Fox J 2001 Calcilytic compounds: potent and selective $\mathrm{Ca}^{2+}$ receptor antagonists that stimulate secretion of parathyroid hormone. Fournal of Pharmacology and Experimental Therapeutics 299 323-331.

Parfitt AM 2001 Parathyroid growth: normal and abnormal. In The Parathyroids, edn 2, pp 293-329. Eds JP Bilezikian, R Marcus \& MA Levine. San Diego, CA: Academic Press.

Seeman E \& Delmus PD 2001 Reconstructing the skeleton with intermittent parathyroid hormone. Trends in Endocrinology and Metabolism 12 281-283.

Wada M, Furuya Y, Sakiyama J, Kobayashi N, Miyata S, Ishii H \& Nagano N 1997 The calcimimetic compound NPS R-568 suppresses parathyroid cell proliferation in rats with renal insufficiency: control of parathyroid cell growth via a calcium receptor. Journal of Clinical Investigation 100 2977-2983.

Received in final form 3 June 2002 Accepted 6 June 2002 